\title{
Evoluzione della tecnica d'innesto su barbatella radicata
}

\author{
R. Perniola, L.R. Forleo e A.D. Marsico \\ CREA Centro di Viticoltura ed Enologia, 70010 Turi-Bari, Italy
}

\begin{abstract}
In viticoltura, per la realizzazione di nuovi impianti sono utilizzate piante bimembri ottenute in vivaio o direttamente in campo. Negli ultimi anni, allo scopo di ottenere rapidamente nuovi impianti, si sta diffondendo la tecnica dell'innesto su barbatella radicata. Tale tecnica, realizzata in vivaio, prevede l'innesto a tavolino delle marze della varietà scelta su barbatelle radicate di portainnesto di specie di Vitis americane. Dopo un periodo di forzatura e ambientamento in vivaio, le piantine vengono messe a dimora in campo, nello stesso anno di realizzazione dell'innesto. Al fine di valutare tale tecnica, presso il CREA Viticoltura ed enologia sede di Turi, è stata sperimentata la possibilità di utilizzare due differenti tipologie d'innesto su diversi portinnesti. Per assicurare una buona resistenza del punto d'innesto ed una buona protezione dalla contaminazione e dalla disidratazione è stato utilizzato un materiale di saldatura costituito da una pellicola elastica e semitrasparente. La sperimentazione è stata condotta per due anni, su 12 varietà di uve da tavola di recente costituzione. I risultati hanno mostrato buoni attecchimenti e saldatura dei bionti. Alcune differenze sono state riscontrate sui portinnesti utilizzati e sui tempi di germogliamento dopo l'innesto.
\end{abstract}

\section{Introduzione}

L'innesto è un' antica tecnica di propagazione vegetativa, realizzata collegando due segmenti di pianta, la parte superiore conosciuta come "nesto" e la parte radicale chiamata "portainnesto". L'innesto, pertanto, può essere definito come la fusione naturale o deliberata di parti di piante così da permettere la continuità vascolare tra loro [1] costituendo un organismo geneticamente composito che sarà funzionante come una pianta singola.

\subsection{Usi dell'innesto}

L'innesto è una pratica che si è sviluppata come tecnica colturale in grado di dare risposte ad alcune criticità. Nel tempo ha avuto un grande impatto su molte pratiche e continua ad essere importante nelle numerose implicazioni in cui è coinvolto. Di seguito un elenco di alcuni dei molti usi dell'innesto: 1) Propagazione vegetativa. 2) Riduzione del periodo giovanile. 3) Cambio di cultivar. 4) Creazione di forme di crescita insolite. 5) Riparazione e/o sostituzione di apparati radicali danneggiati. 6) Controllo della vigoria. 7) Resistenze a stress biotici. 8) Adattamento a condizioni del suolo limitanti. 9) Accertamento della presenza /assenza di agenti infettivi. 10) Studi fisiologici. 11) Incremento rapido della produzione di viti di nuove varietà o di varietà rare $[2,3]$.

\subsection{L'innesto in viticoltura}

La conoscenza della tecnica di innesto in arboricoltura risale a tempi antichissimi. Numerose testimonianze riportate da greci e latini citano l'innesto nella pratica viticola. Alcuni esempi sono riportati da: Teofrasto; Catone; Columella; Plinio; Varrone; Virgilio e Palladio.
Per poi continuare con scrittori rinascimentali come Pietro de Crescenzi; Herrera; Giovan Battista Porta; Ludovico Ottelio [4].

Nella seconda metà del $19^{\circ}$ secolo l'innesto della vite è diventato pratica indispensabile per la realizzazione di nuovi impianti, in quanto in grado di contrastare gli effetti della fillossera (Daktulosphaira vitifoliae Fitch) che fu inavvertitamente introdotta in Europa e che distrusse gradualmente i vigneti europei [5]. I portinnesti furono selezionati da alcune specie di Vitis spp originarie del Nord americana, e più tardi furono realizzati ibridi tra le diverse specie per combinare al meglio le specifiche caratteristiche di ogni specie. In questo modo è stato possibile ottenere portinnesti resistenti/tolleranti, oltre che alla fillossera, anche ad altri fattori biotici come i nematodi e a condizioni del suolo limitanti la coltivazione della vite (fattori abiotici), come la presenza di ristagni idrici, la scarsa disponibilità idrica o l'eccesiva presenza di calcare attivo (terreni clorosanti).

\subsection{Classificazione}

Le variabili che intervengono nella realizzazione degli innesti riguardano: il luogo in cui viene compiuto, la tecnica, lo stato vegetativo delle viti e l'epoca. In riferimento al luogo in cui viene realizzato è possibile classificare gli innesti in campo o a tavolino. Gli innesti in campo si dicono tali quando realizzati su piante portinnesto messe a dimora l'anno prima, mentre a tavolino quando realizzati in vivaio nella preparazione delle talee-innesto radicate. In riferimento alla tecnica, abbiamo gli innesti a spacco, a doppio spacco, alla maiorchina, ad omega, a "V", a incastro, ecc. In funzione dello stato vegetativo, innesti a gemma dormiente o a gemma vegetante (innesti 
Tabella 1. Descrizione delle 12 varietà utilizzate come nesto.

\begin{tabular}{|l|c|c|c|c|}
\hline Nome & $\begin{array}{c}\text { Cotore } \\
\text { degli } \\
\text { acini }\end{array}$ & $\begin{array}{c}\text { Peso } \\
\text { acino } \\
(\mathbf{g})\end{array}$ & $\begin{array}{c}\text { Peso } \\
\text { grappolo } \\
(\mathbf{g})\end{array}$ & $\begin{array}{c}\text { Pruduzione } \\
\text { potenziale } \\
(\mathbf{q} / \mathbf{h a})\end{array}$ \\
\hline Appia & Nera & 4.5 & 400 & 320 \\
\hline Barese & Nera & 5.0 & 660 & 720 \\
\hline Barolum & Nera & 4.0 & 300 & 220 \\
\hline Daunia & Rossa & 4.5 & 400 & 400 \\
\hline Egnazia & Nera & 5.0 & 600 & 560 \\
\hline Japigia & Bianca & 6.0 & 630 & 480 \\
\hline Maula & Nera & 6.0 & 260 & 240 \\
\hline Netium & Rossa & 6.0 & 420 & 500 \\
\hline Norba & Rosa & 5.0 & 600 & 640 \\
\hline Peucetia & Bianca & 3.5 & 450 & 320 \\
\hline Siris & Nera & 4.5 & 450 & 320 \\
\hline Vigilarium & Bianca & 4.0 & 320 & 320 \\
\hline
\end{tabular}

erbacei). Per quanto riguarda l'epoca questi possono essere, invernali, primaverili ed estivi.

\subsection{L'innesto nelle uve da tavola}

In Italia, ed in Puglia in particolare, dove è concentrato il $60 \%$ della produzione di uva da tavola, i nuovi impianti sono realizzati mettendo a dimora gli ibridi portinnesto e nell'anno successivo vengono poi innestati in campo, con la varietà desiderata. Questo nel periodo invernaleprimaverile. Meno diffusa è la realizzazione di vigneti con barbatelle già innestate (derivanti da innesti talea realizzati in vivaio).

Negli ultimi anni si sta diffondendo la tecnica dell'innesto su barbatella radicata. In casi particolari, allo scopo di disporre immediatamente della varietà voluta, si può operare un innesto su barbatella franca dell'età di un anno. Dopo aver rinforzato il punto d'innesto con apposito nastro elastico, si paraffina per evitare la disidratazione e si mette immediatamente a dimora in vigneto. Con le dovute cure si possono ottenere buoni attecchimenti, con uno sviluppo vegetativo rilevante già al primo anno [6].

\subsection{Sperimentazione proposta}

Per valutare le possibilità offerte da questa nuova tecnica nella propagazione di nuove varietà [7], recentemente ottenute presso il CREA Viticoltura ed Enologia di Turi, è stata realizzata una prova di confronto utilizzando differenti tipologie d'innesto e portinnesti.

\section{Materiali e metodi}

\subsection{Condizioni sperimentali}

La sperimentazione è stata condotta su materiale vegetale raccolto nel 2017 e nel 2018. Tralci legnosi di Vitis vinifera di 12 nuove varietà sono state raccolti durante la fase fenologica di "riposo vegetativo" e conservati in cella frigo alla temperatura di $+4{ }^{\circ} \mathrm{C}$ e umidità relativa prossima al 90\%. In Tabella 1 sono riportate le principali caratteristiche delle 12 varietà impiegate nella sperimentazione:

Nel mese di febbraio dai tralci legnosi sono state raccolte le gemme che sono state innestate su barbatelle portainnesto radicate, "certificate" secondo la Direttiva 2002/11/CE, delle seguenti varietà: $140 \mathrm{Ru}$ (incrocio $\mathrm{V}$. berlandieri $\times \mathrm{V}$. rupestris), $1157.11 \mathrm{C}$ (incrocio $\mathrm{V}$. berlandieri $\times$ V. riparia), 1103P (incrocio V. berlandieri $\times$ $\mathrm{V}$. rupestris) e $110 \mathrm{R}$ (incrocio $\mathrm{V}$. berlandieri $\times \mathrm{V}$. rupestris) [8].

Sono stati eseguiti due differenti tipologie di innesto: "a spacco", con taglio del portainnesto e sagoma manuale del nesto e "ad omega", con taglio del nesto e del portainnesto eseguito meccanicamente. Per assicurare la stabilità e la protezione dell'innesto è stato utilizzato una pellicola elastica, semitrasparente, progettata per sigillare ermeticamente e proteggere dalla disidratazione gli innesti. La pellicola è estensibile e sigilla perfettamente anche aree e forme irregolari.

Le piante innestate sono state trasferite in vasi dalle dimensioni di $10 \mathrm{~cm} \times 10 \mathrm{~cm}$ con un'altezza di $25 \mathrm{~cm}$, riempiti con terriccio e perlite nelle proporzioni di 3:2. Le piantine sono state quindi trasferite in un locale riscaldato alla temperatura di $28^{\circ} \mathrm{C}$, in condizioni di umidità relative pari al 90-95\%, dove sono state conservate per un periodo di circa $15 \mathrm{gg}$. Successivamente le piante sono state sottoposte a due fasi di ambientamento: la prima per circa 7 gg. a $20^{\circ} \mathrm{C}$ e la seconda, prima del trasferimento in campo, per circa $15 \mathrm{gg}$. alla temperatura di $16^{\circ} \mathrm{C}$ ed umidità relativa del $70 \%$.

Per ciascuna combinazione nesto/portainnesto/tipo di innesto sono state realizzate un numero variabile di piante (da 10 a 24), a 30-60 giorni dal trasferimento in campo è stata valutata la percentuale di germogliamento, mediante la seguente formula:

Germogl. $\%=($ n. di piante germogliate/n. di piante totali) $\times 100$

\subsection{Analisi statistica}

Preliminarmente i dati sono stati sottoposti a test di Shapiro-Wilk e test di Levene, al fine di verificarne rispettivamente la normalità della distribuzione e l'omogeneità delle varianze.

Su $140 \mathrm{Ru}$, mediante il test di Wilcoxon della somma dei ranghi, è stato valutato l'effetto del tipo di innesto impiegato (spacco/omega) sulla percentuale di germogliamento, indipendentemente dalla varietà di nesto impiegata. Nell'ambito dell'innesto ad omega, il medesimo test statistico è stato impiegato, per valutare l'effetto di diversi portainnesti (140 Ru/157.11 C) sulla percentuale di germogliamento, indipendentemente dalla varietà utilizzata. Infine, nell'ambito dell'innesto "a spacco", è stata valutato l'effetto di tre diversi portainnesti (140 Ru, 110 R e $1103 \mathrm{P})$ sulla percentuale di germogliamento delle 12 varietà impiegate, mediante il test non parametrico di Kruskall-Wallis.

Tutte le analisi statistiche sono state eseguite mediante il software statistico R (versione 3.4).

\section{Risultati}

Durante il periodo di ambientamento le viti innestate sono state monitorate per rilevare l'inizio del germogliamento e valutare la velocità di attecchimento degli innesti. Durante e dopo il periodo di ambientamento su tutte le piantine è stato rilevato il numero di viti germogliate e lo stadio 
Tabella 2. Influenza della tipologia d'innesto su $140 \mathrm{Ru}$.

\begin{tabular}{|c|c|c|c|}
\hline $\begin{array}{c}\text { Tipologia } \\
\text { d'innesto }\end{array}$ & $\begin{array}{c}\text { Innesti } \\
\text { attecchiti }\end{array}$ & $\begin{array}{c}\text { Innesti non } \\
\text { attecchiti }\end{array}$ & \% di germogliamento \\
\hline Omega & 188 & 52 & 78.3 \\
\hline Spacco & 209 & 55 & 80.9 \\
\hline
\end{tabular}

Tabella 3. Influenza del portinnesto su innesto ad omega.

\begin{tabular}{|c|c|c|c|}
\hline Portinnesto & $\begin{array}{c}\text { Innesti } \\
\text { attecchiti }\end{array}$ & $\begin{array}{c}\text { Innesti non } \\
\text { attecchiti }\end{array}$ & \% di germogliamento \\
\hline $140 \mathrm{Ru}$ & 188 & 52 & 78.3 \\
\hline $157.11 \mathrm{C}$ & 60 & 60 & 50.0 \\
\hline
\end{tabular}

Tabella 4. Influenza del portinnesto su innesto a spacco.

\begin{tabular}{|c|c|c|c|}
\hline Portinnesto & $\begin{array}{c}\text { Innesti } \\
\text { attecchiti }\end{array}$ & $\begin{array}{c}\text { Innesti non } \\
\text { attechiti }\end{array}$ & \% di germogliamento \\
\hline $140 \mathrm{Ru}$ & 120 & 24 & 80.9 \\
\hline $1103 \mathrm{P}$ & 141 & 3 & 97.9 \\
\hline $110 \mathrm{R}$ & 209 & 55 & 83.3 \\
\hline
\end{tabular}

di sviluppo dei germogli. Successivamente, durante la fase di sviluppo delle viti in campo, è stata rilevata la lunghezza dell'intero germoglio ed il diametro dei germogli al secondo internodo basale (dati non mostrati in questo lavoro).

\subsection{Attecchimenti}

In Tabella 2 sono riportati i dati elaborati del numero di innesti e le percentuali di germogliamento ottenute in funzione della metodica d'innesto impiegata (spacco/omega).

In particolare, le 12 varietà saggiate innestate sul portinnesto $140 \mathrm{Ru}$ mediante la tecnica dell'innesto "a spacco", hanno mostrato una percentuale di germogliamento significativamente più elevata $(p=0.002337)$, in confronto a quella ottenuta impiegando la tecnica dell'innesto "a omega".

In Tabella 3 sono riportate le percentuali di germogliamento ottenute impiegando la tecnica dell'innesto "a omega" su due differenti portainnesti (140 Ru e 157.11 C).

Indipendentemente dalla varietà del nesto impiegata, nell'ambito dell'innesto ad omega, il $140 \mathrm{Ru}$ risulta essere significativamente $(p=0.001139)$ più performante $(78.3 \%)$ rispetto al $157.11 \mathrm{C}(50.0 \%)$.

In Tabella 4 sono riportate le percentuali di germogliamento ottenute complessivamente con le 12 varietà, su tre differenti portainnesti (140 Ru, $1103 \mathrm{P}$ e $110 \mathrm{R})$.

Nell' ambito degli innesti realizzati "a spacco" i diversi portinnesti hanno mostrato un buon attecchimento, e fra tutti il $1103 \mathrm{P}$ ha garantito un attecchimento pari al $97.9 \%$, seguito dal $110 \mathrm{R}(83.3 \%)$ e $140 \mathrm{Ru}(79.2 \%)$. Tuttavia, le differenze di performance tra i tre portainnesti saggiati, non sono risultate statisticamente significative $(p=0.07347)$.

Infine, anche le diverse varietà saggiate hanno mostrato in maniera differente l'attechimento dei due bionti (Tabella 5).

Particolarmente interessanti, sono risultate le varietà Barese, Maula e Netium che superano 1'89\% di attecchimento, mentre altre hanno manifestato percentuali
Tabella 5. Influenza della varietà sull'attecchimento dei nesti.

\begin{tabular}{|c|c|c|c|}
\hline Varietà & $\begin{array}{c}\text { Innesti } \\
\text { attechiti }\end{array}$ & $\begin{array}{c}\text { Innesti non } \\
\text { attecchiti }\end{array}$ & \% di germogliamento \\
\hline Appia & 62 & 16 & 81.5 \\
\hline Barese & 70 & 8 & 85.0 \\
\hline Barolum & 66 & 12 & 82.3 \\
\hline Daunia & 56 & 10 & 85.7 \\
\hline Egnazia & 67 & 11 & 87.3 \\
\hline Japigia & 43 & 35 & 62.0 \\
\hline Maula & 70 & 8 & 86.0 \\
\hline Netium & 59 & 7 & 87.7 \\
\hline Norba & 64 & 14 & 79.2 \\
\hline Peucctia & 45 & 33 & 53.8 \\
\hline Siris & 56 & 22 & 72.8 \\
\hline Vigilarium & 60 & 18 & 73.8 \\
\hline
\end{tabular}

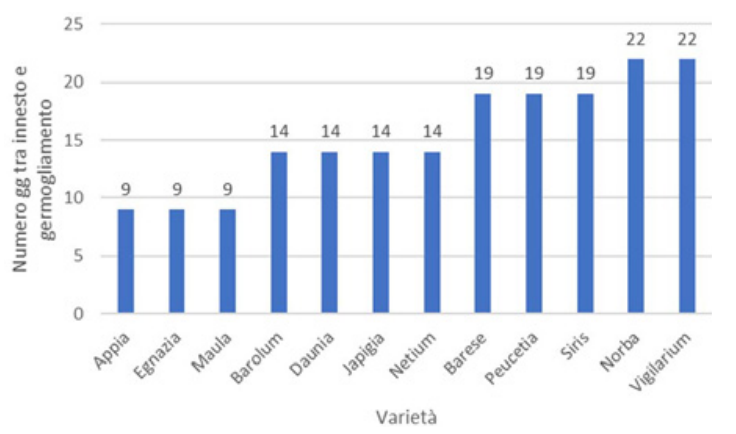

Figura 1. Velocità di attecchimento delle 12 varietà.

di attecchimento inferiori al 57\% come le varietà Japigia e Peucetia. Tali differenze, tuttavia, non possono essere considerate statisticamente differenti $(p=0.1527)$

\subsection{Velocità di attecchimento}

Durante il periodo di forzatura e acclimatamento le viti innestate sono state monitorate, e per ogni varietà sono state rilevate le date di inizio germogliamento.

Questo rilievo ha permesso di valutare il numero di giorni intercorsi fra l'innesto ed il germogliamento delle viti così come riportato in Fig. 1. Il grafico mostra che le varietà Appia, Maula ed Egnazia abbiano tempi brevi di attecchimento pari a $9 \mathrm{gg}$., altre anno un comportamento intermedio ed altre ancora come la Norba e Vigilarium, presentano tempi più lunghi e pari a $22 \mathrm{gg}$.

\section{Conclusioni e sviluppi futuri}

La tecnica dell'innesto, su barbatelle di un anno radicate, ha permesso di ottenere in maniera rapida piante bimembri da poter trasferire in campo già al primo anno. Alla fine del primo anno sono state ottenute piante ben sviluppate e potenzialmente produttive già al secondo anno dall'impianto.

Prendendo in considerazione la tecnica dell'innesto impiegata, l'innesto "a spacco" è risultato essere più performante rispetto a quello "a omega").

Mentre nell' ambito dell'innesto ad omega, le 12 varietà saggiate nel loro complesso, si sono adattate meglio al $140 \mathrm{Ru}$ piuttosto che al $157.11 \mathrm{C}$. Nell' ambito dell'innesto 
a spacco, nessuna differenza statisticamente significativa è stata riscontrata riguardo gli effetti sulla percentuale di attecchimenti sottesi ai tre portainnesti e alle 12 nuove varietà prese in esame. Le percentuali di attecchimento più basse potrebbero essere state condizionate da fattori d'incompatibilità fra nesto e portinnesto [9] che spesso pregiudicano la buona riuscita dell'impianto di un nuovo vigneto.

Pertanto, in un prossimo futuro sarà necessario valutare le combinazioni d'innesto fra le nuove varietà e portinnesti con le tecniche più diffuse di innesto realizzato in campo ed innesto-talea realizzato in vivaio. Durante la sperimentazione, per la saldatura degli innesti, è stato possibile testare una pellicola elastica che sembra garantire un'ottima stabilità ed una buona protezione dalla disidratazione, senza la necessaria paraffinatura che altri materiali prevedono. Pertanto, in un prossimo futuro tali materiali saranno confrontati con quelli solitamente utilizzati per testarne le effettive performance. Le attività, proseguiranno anche nella definizione delle necessarie cure colturali in grado di permettere un più uniforme sviluppo delle piante dopo il trasferimento in campo.

\section{Bibliografia}

[1] P. Pina, P. Errea, Scientia Hort. 106, 1 (2005)

[2] A.J. Winkler, General Viticulture (University California Press, 1974)

[3] K. Mudge, et al., Horticultural Rev. 35, 9 (2009)

[4] G. Venturi, Trattato Degli Innesti (Davolio, 1816)

[5] J. Granett, et al., Ann. Rev. Entomol. 46, 387 (2001)

[6] Morando, et al., Vigna nuova. Edizioni VitEn, Calosso - Asti (Italy) (2016), p. 77

[7] Perniola, et al., Rivista di Frutticoltura e di Ortofloricoltura 83, 18 (2019)

[8] A.A.V.V. Registro nazionale varietà di vite, portinnesti. Mipaaft. (2019)

[9] H.T. Hartman, D.E. Kester, F.T. Davies, R. Geneve, Plant Propagation (Prentice-Hall, Englewood Cliffs, NJ, 2002) 